\title{
Correlation of PMN elastase and PMN elastase-to-neutrophil ratio with disease activity in patients with myositis
}

\author{
Siyu Wu, Wanchan Peng, Yunli Zhang, Jingjing Guo, Jinfang Fu and Wei Wang * (1)
}

\begin{abstract}
Background: Polymorphonuclear (PMN) elastase plays an important role in a variety of inflammatory disorders. Our aim was to analyse PMN elastase in idiopathic inflammatory myopathies (IIMs) and its association with disease activity.

Methods: PMN elastase levels were measured using enzyme-linked immunosorbent assay in serum samples obtained from 74 patients with myositis (58 with dermatomyositis [DM] and 16 with polymyositis [PM]) and 22 healthy controls. Receiver operating characteristic (ROC) curve analysis was performed to evaluate the discriminant capacity of PMN elastase level and PMN elastase-to-neutrophil ratio (ENR) in patients with active and remission myositis. The association of serum PMN elastase level and ENR with disease variables was evaluated in patients with IIMs. The disease specificity of PMN elastase level and ENR was further examined in 60 patients with other systemic autoimmune diseases.
\end{abstract}

Results: PMN elastase level and ENR were significantly higher in patients with active IIMs, DM, and PM than in patients with remission. ROC curve analysis revealed that PMN elastase level and ENR both outperformed creatine kinase (CK), the currently used laboratory marker, and strongly discriminated patients with active disease and those with remission of IIMs, DM, and PM (area under the ROC curve [AUC] 0.9, 0.9, and 0.88 for PMN elastase; AUC 0.96, 0.96, and 1.0 for ENR; AUC $0.72,0.70$, and 0.80 for CK, respectively). PMN elastase level and ENR were positively correlated with myositis disease activity assessment, CK, lactate dehydrogenase, aspartate aminotransferase, alanine aminotransferase, C-reactive protein, and erythrocyte sedimentation rate. PMN elastase level and ENR were higher in the anti-PM$\mathrm{Scl}$ positive myositis group than those in the anti-PM-Scl negative myositis group. Nevertheless, PMN elastase was not a specific disease marker for IIMs when compared with other autoimmune diseases.

Conclusions: PMN elastase, particularly ENR, were significantly correlated with disease activity and could serve as useful biochemical markers for evaluating the disease activity of patients with IIMs. Thus, they are potentially helpful in monitoring disease progression and guiding treatment.

Keywords: PMN elastase, PMN elastase-to-neutrophil ratio, Idiopathic inflammatory myopathies, Disease activity

\section{Background}

Idiopathic inflammatory myopathies (IIMs), including dermatomyositis (DM), polymyositis (PM), inclusion body myositis, and immune-mediated necrotising

\footnotetext{
*Correspondence: weiweiwang1983@163.com

Department of Clinical Laboratory, Xiangya Hospital, Central South

University, 87 Xiangya Road, Changsha, Hunan, People's Republic of China
}

myopathy, which have been recently distinguished [1-3], are rare systemic autoimmune diseases. They are characterised by chronic proximal muscle inflammation and weakness, and/or skin rash. The serological features include a high titration of serum autoantibodies and muscle enzymes, and the histological features are infiltration of mononuclear cells and inflammatory cells in the skeletal muscle tissue [1, 4]. Inflammatory cell infiltration 
in muscle tissue may contribute to the muscle damage and dysfunction by releasing cytokines, cytotoxic molecules, or proteinase, leading to disease progression.

Disease activity of IIMs is currently assessed using muscle enzyme testing and clinical evaluation. Muscle enzyme testing includes serum creatine kinase (CK), aspartate aminotransferase (AST), alanine aminotransferase (ALT) and lactate dehydrogenase (LDH) activity assessment and has been shown to moderately correlate with disease activity in IIMs [5, 6]. However, clinical evaluation depends on the experience of health care professionals and patient collaboration $[7,8]$. Thus, identifying objective tools or biomarkers is urgent for monitoring IIM disease activity. Recently, longitudinal cohort and multi-cohort validation studies have revealed that galectin-9 and CXCL-10 are sensitive and reliable biomarkers for disease activity in juvenile DM $[9,10]$. In adult patients with myositis, several studies revealed that S100A11 [11], serum-soluble TRAIL [12], YKL-40 [13], and anti-EJ [14] are correlated with disease activity, but there is a lack of further research on their diagnostic value. Hence, objective biomarkers for monitoring disease activity in adult patients with myositis need further investigation.

Polymorphonuclear (PMN) leukocytes or neutrophils are inflammatory cells that play an important role as primary defence cells in the inflammatory response against invading pathogens or damaged tissue, by using proteinases. One of these proteinases is PMN elastase, which is localised in the azurophilic granules. The activity of PMN elastase is regulated by proteinase inhibitors, such as $\alpha 1$-proteinase inhibitor [15]. An overwhelming release of PMN elastase, which exceeds the inhibitory potential of the proteinase inhibitor, can lead to active PMN elastase persisting. In combination with simultaneously produced oxidants $\left(\mathrm{O}_{2}\right.$-radicals, $\mathrm{H}_{2} \mathrm{O}_{2}, \mathrm{OH}$-radicals $)$, this can cause local tissue injury. Previous studies showed that PMN elastase plays an important role in a variety of inflammatory disorders [16-19]. Most studies have demonstrated a clear correlation between PMN elastase and clinical course of the disease, and PMN elastase may be a biochemical marker reflecting the intensity of inflammation in patients with inflammatory diseases [20-22]. Moreover, studies on PMN elastase and IIMs showed that neutrophil serine proteinases, including cathepsin G, PMN elastase, and proteinase 3 , are upregulated in patients with IIMs and increase the permeability of vascular endothelial cells to permit infiltration of inflammatory cells into the muscle $[23,24]$. However, the role of PMN elastase in evaluating the disease activity of patients with IIM remains unknown and needs further investigation. Thus, the major aim of this study was to evaluate serum PMN elastase levels in patients with IIMs and other autoimmune diseases, with an emphasis on investigating the role of PMN elastase to distinguish between patients with active IIMs and those in remission.

\section{Methods \\ Human subjects}

The study group comprised 74 patients with myositis (58 with DM, 16 with PM). Patients with PM and DM fulfilled the Bohan and Peter diagnostic criteria $[25,26]$ and had no other systemic autoimmune diseases, infections, or major illnesses. Disease activity was assessed using the myositis disease activity assessment (MYOACT), established by the International Myositis Assessment and Clinical Studies group [27]. This tool was proved to be reliable for evaluating disease activity in Chinese patients with DM or PM [28]. Active disease was defined by clinicians based on clinical criteria, including typical skin manifestations, muscle involvement, and/or an increase in muscle enzyme levels. The patients with myositis in this study were also suffering from arthritis (30\%), dysphagia $(2.7 \%)$, or ulceration $(2.7 \%)$. In addition, $43 \%$ of the patients with myositis were diagnosed with interstitial lung disease (ILD) and $2.7 \%$ with cardiac involvement. ILD was identified using high-resolution computed tomography.

The control group included healthy controls ( $\mathrm{HCs}$, $\mathrm{n}=22$ ) matched for age and sex with the patients. To determine the disease specificity of the biomarkers, different disease controls were added to the study: systemic lupus erythematosus (SLE, $\mathrm{n}=20$ ), systemic sclerosis (SSc, $\mathrm{n}=20)$, and rheumatoid arthritis (RA, $\mathrm{n}=20$ ). Patients with RA were diagnosed according to the American College of Rheumatology/European League against Rheumatism classification criteria. Diagnosis of SLE and SSc were performed based on the American College of Rheumatology criteria. Active disease was assessed mainly by clinicians and defined as having an SLE disease activity index score $\geq 4$ for SLE [29], the European Scleroderma Trails and Research group activity index score $>2.5$ for SSc [30], and a disease activity score $28 \geq 3.2$ for RA [31].

Informed written consent was obtained from all subjects, and the study was approved by the Ethics Committee of Xiangya Hospital, Central South University, where the study was performed.

\section{Laboratory analysis}

The following data for patients with IIMs were additionally collected: CK, LDH, ALT, AST, erythrocyte sedimentation rate (ESR), C-reactive protein (CRP), complement fractions (C3 and $\mathrm{C} 4$ ), neutrophil and lymphocyte counts, and the ratio of neutrophil to lymphocyte (NLR). These were all analysed using routine laboratory 
techniques within $24 \mathrm{~h}$ after enrolment. Antinuclear antibody (ANA) was detected by indirect immunofluorescence using Hep-2 cells (Euroimmun, Lübeck, Germany). Myositis-specific and -associated autoantibodies were detected using immunoblotting (Euroimmun, Lübeck, Germany). The demographic data for the disease groups are described in Table 1.

\section{Measurement of serum PMN elastase levels}

Serum samples were stored at $-20{ }^{\circ} \mathrm{C}$ until analysis. Serum levels of human PMN elastase were measured using a commercially available enzyme-linked immunosorbent assay kit (Abcam, Cambridge, MA). Standards and patient samples were analysed in duplicates according to the manufacturer's instructions.

\section{Statistical analysis}

Data were tested for normality using KolmogorovSmirnov test in the total sample and within each group of patients (DM and PM). Differences between groups were assessed with the Mann-Whitney U test. Since most of the variables were not normally distributed, data are shown as medians (min-max) or medians (interquartile range). The correlations between variables were evaluated using Spearman's rank correlation. Receiver operating characteristic (ROC) curves were used to evaluate the significance of PMN elastase levels to distinguish between myositis patients with active disease and those in remission. The Youden index was calculated as sensitivity + specificity -1 . The best critical point was selected as the largest tangential point of the Youden index. A P value $<0.05$ was used to indicate a statistically significant result. Statistical analysis was performed using SPSS 20.0 (IBM SPSS Statistics, IBM Corporation, Chicago, IL, USA) or Prism software v. 5.0 (GraphPad Software, Inc., San Diego, CA).

\section{Results \\ Serum levels of PMN elastase in patients with myositis and controls}

The concentrations of PMN elastase in patients with myositis and HCs are shown in Fig. 1a. PMN elastase levels in patients with IIMs were particularly upregulated compared with those in HCs (1521.8 (787.4-3820.5) vs 1193.6 (518.8-1513.9), $\mathrm{ng} / \mathrm{mL}$, median (interquartile range); $\mathrm{P}=0.019$ ). After dividing the patients with IIMs into subgroups, only those with DM showed significantly higher elevation of PMN elastase when compared with HCs (1569.6 (871.1-4676.0) vs 1193.6 (518.81513.9), $\mathrm{ng} / \mathrm{mL}, \mathrm{P}=0.008$; Fig. 1a). No significant difference was found between patients with PM and HCs

Table 1 Clinical characteristics of the study subjects

\begin{tabular}{|c|c|c|c|c|c|c|}
\hline & IIMs & DM & PM & SLE & SSc & RA \\
\hline Number & 74 & 58 & 16 & 20 & 20 & 20 \\
\hline Gender (female/male) & $55 / 19$ & $41 / 17$ & $14 / 2$ & $17 / 3$ & $12 / 8$ & $18 / 2$ \\
\hline Age (years) & $50(20-75)$ & $50(20-75)$ & $51(32-69)$ & $41(14-62)$ & $57(14-73)$ & $58(44-70)$ \\
\hline Disease durations (years) & $0.8(0.1-12)$ & $0.8(0.1-12)$ & $0.7(0.1-4)$ & $3.5(0.1-15)$ & $2(0.7-7)$ & $1(0.1-10)$ \\
\hline Active/remission (number) & $44 / 30$ & $35 / 23$ & $9 / 7$ & $10 / 10$ & $10 / 10$ & $10 / 10$ \\
\hline MYOACT & $1.4(0.7-4.2)$ & $1.4(0.7-4.2)$ & $1.4(0.7-2.6)$ & - & - & - \\
\hline \multicolumn{7}{|l|}{ Muscle enzymes } \\
\hline CK, uKat/L & $59(12.5-6621)$ & $58(12.5-6621)$ & $65.1(21.3-5890.8)$ & - & - & - \\
\hline AST, uKat/L & $30.8(11.2-420.1)$ & $30.8(12.4-420.1)$ & $29.8(11.2-227.6)$ & $21.7(13.5-68.9)$ & $25.1(13.5-66.3)$ & $17.3(9.6-45.2)$ \\
\hline ALT, uKat/L & $29.9(6.2-201.8)$ & 31.7 (10.4-201.8) & $25.7(6.2-139)$ & $19(9.6-152.2)$ & $14.3(3.6-72.3)$ & $13.4(4.9-59.9)$ \\
\hline LDH, uKat/L & $284(137-1267)$ & $282.5(141-1267)$ & $286(137-767)$ & - & - & - \\
\hline \multicolumn{7}{|l|}{ Inflammation marker } \\
\hline $\mathrm{CRP}, \mathrm{mg} / \mathrm{L}$ & $3.2(1-165)$ & $3.8(1.1-165)$ & $2.5(1-7.1)$ & $5.1(1.3-242)$ & $4.1(1-43.2)$ & $14.1(1.5-86.5)$ \\
\hline $\mathrm{ESR}, \mathrm{mm} / \mathrm{h}$ & $49(4-120)$ & $53(4-120)$ & $30(6-85)$ & $55(2-120)$ & $54.5(2-114)$ & $92.5(33-120)$ \\
\hline $\mathrm{C} 4, \mathrm{mg} / \mathrm{L}$ & 203.5 (39.6-454) & $222(39.6-454)$ & 185 (102-437) & $153(23.2-365)$ & $188(108-375)$ & $208(117-318)$ \\
\hline $\mathrm{C} 3, \mathrm{mg} / \mathrm{L}$ & $836(415-1690)$ & $826(415-1690)$ & 803 (566-955) & $704(81-1210)$ & 795 (411-1410) & $1010(817-1220)$ \\
\hline Neutrophil, $\times 10^{9} / \mathrm{L}$ & $4.5(1.4-15.1)$ & $5.2(1.4-12.4)$ & $4.7(1.5-15.1)$ & $4.8(0.7-11.8)$ & $4.4(1.8-11.5)$ & $4.5(1.9-8.6)$ \\
\hline Lymphocyte, $\times 10^{9} / \mathrm{L}$ & $1.4(0.1-4.1)$ & $1.3(0.1-4.1)$ & $1.4(0.3-3.7)$ & $1.4(0.1-2.8)$ & $1.3(0.6-2.6)$ & $1.6(0.9-3.1)$ \\
\hline NLR & $3.7(0.7-23)$ & $4.4(1.3-23)$ & $3.9(0.8-14)$ & $3.6(1.2-31)$ & $3.3(2.1-11.5)$ & $3.0(1.4-7.9)$ \\
\hline
\end{tabular}

Data are presented as number of patients or median (min-max)

MYOACT myositis disease activity assessment, CK creatine phosphokinase, AST aspartate aminotransferase, $A L T$ alanine aminotransferase, $L D$ lactate dehydrogenase, $C R P$ C-reactive protein, ESR erythrocyte sedimentation rate, $C 4$ complement fraction $4, C 3$ complement fraction $3, N L R$ the ratio of neutrophil count to lymphocyte count 

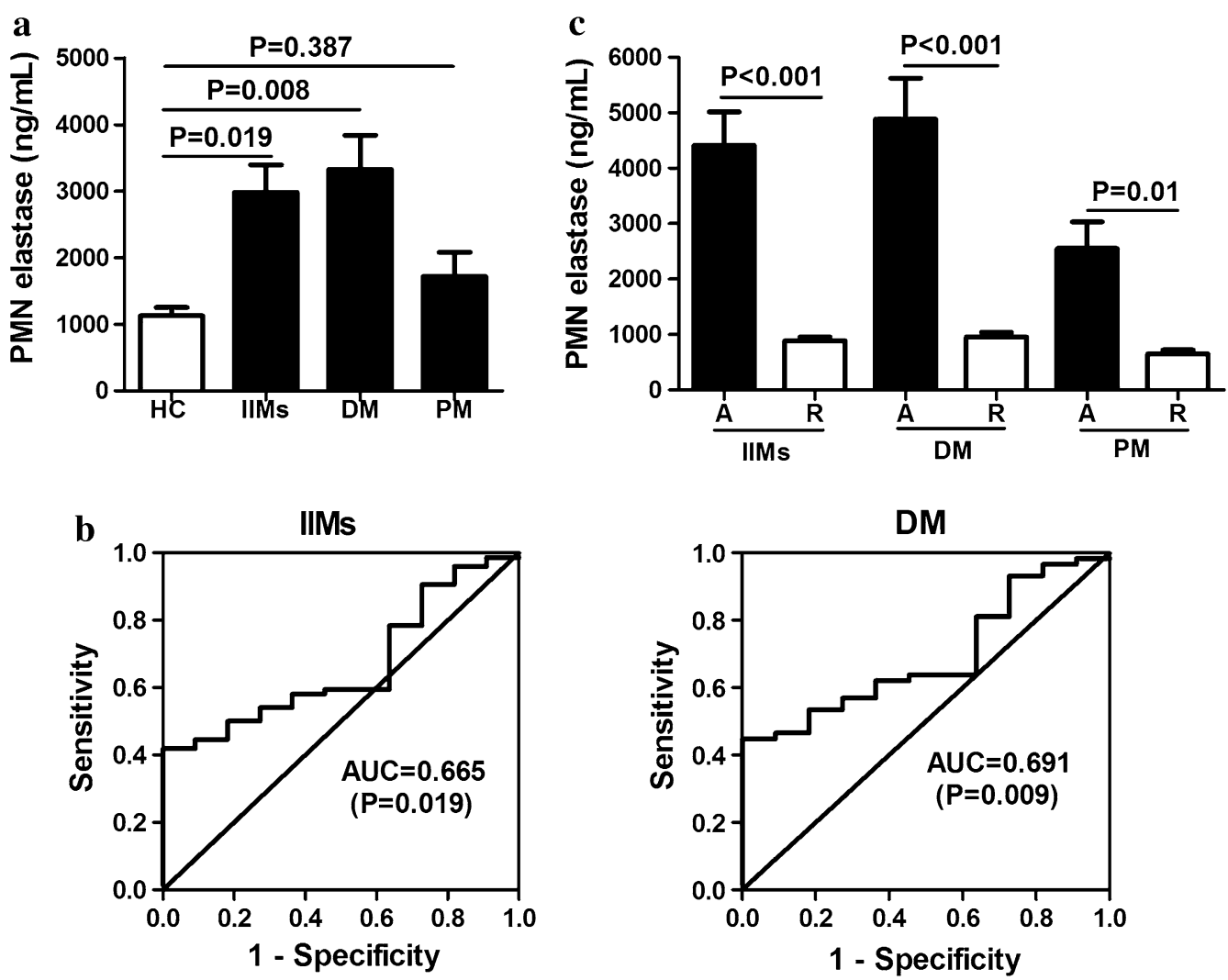

Fig. 1 Serum levels of polymorphonuclear (PMN) elastase in patients with myositis. PMN elastase levels are elevated in patients with idiopathic inflammatory myopathies (IIMs) and dermatomyositis (DM), but not in those with polymyositis (PM) when compared with healthy controls (HCs) (a). Area under receiver operating characteristic (ROC) curve analysis was used to assess the ability of PMN elastase to distinguish among IIMs, DM, and HCs (b). The reference curve is also shown. PMN elastase levels were significantly higher in patients with IIMs, DM, and PM with active disease [A] than those in remission $[\mathrm{R}](\mathbf{c})$

(999.7 (581.4-3204.5) vs 1193.6 (518.8-1513.9), ng/mL, $\mathrm{P}=0.387$; Fig. 1a).

The predictive values of PMN elastase in patients with IIMs and DM versus those in HCs were studied using univariate ROC analysis. The univariate areas under the curve (AUC) for PMN elastase were 0.665 (95\% CI 0.550.78 ) for discriminating between patients with IIMs and HCs ( $\mathrm{P}=0.019$; Fig. $1 \mathrm{~b})$ and 0.691 (95\% CI 0.58-0.81) for discriminating between patients with DM and HCs $(\mathrm{P}=0.009$; Fig. 1b).

\section{Correlation of PMN elastase levels with disease activity}

To investigate the correlation of PMN elastase levels with disease activity, we initially compared IIM patients with active disease and those in remission. PMN elastase levels were significantly higher in patients with active disease than those in remission (3382.9 (1722.5-5821.6) vs 799.8 (556.7-1269.1), ng/mL, $\mathrm{P}<0.001)$; Fig. 1c). After dividing the total patients into DM and PM, both patients with active DM and PM showed higher PMN elastase levels than those in remission (3413.5 (1891.9-5860.0) vs 875.2
(695.2-1425.0), and $2612.6(1258.1-3705.9)$ vs 619.8 (449.8-806.3), ng/mL, $\mathrm{P}<0.001$ and $\mathrm{P}=0.01$; Fig. $1 \mathrm{c}$ ).

To further assess the discriminative power of PMN elastase for active disease and remission, univariate ROC analysis was performed. Comparing active disease and remission, PMN elastase had an AUC of 0.90 for IIMs, 0.90 for DM, and 0.88 for PM (Fig. 2a and Table 2). Based on the coordinates of the ROC curves, the cut-off values of PMN elastase were $1632.4 \mathrm{ng} / \mathrm{mL}$ for IIMs, $1779.0 \mathrm{ng} /$ $\mathrm{mL}$ for DM, and $999.7 \mathrm{ng} / \mathrm{mL}$ for PM, which resulted in a sensitivity of $79 \%, 80 \%$, and $80 \%$ and a specificity of $100 \%$ for IIMs, DM and PM, respectively (Table 2). Moreover, PMN elastase performed better than the current standard laboratory marker CK in patients with myositis (AUC of CK: 0.72 in IIMs, 0.70 in DM, 0.80 in PM).

Moreover, we investigated the correlation of PMN elastase level with disease activity scoring by using the MYOACT tool. Notably, the PMN elastase level was positively correlated with MYOACT, with $\mathrm{r}$ values of 0.549 in IIMs ( $\mathrm{P}<0.001$; Fig. 2b), 0.543 in $\mathrm{DM}(\mathrm{P}<0.001$; Fig. 2b), and 0.506 in $\mathrm{PM}(\mathrm{P}<0.05$; Fig. $2 \mathrm{~b})$. Overall, these data 


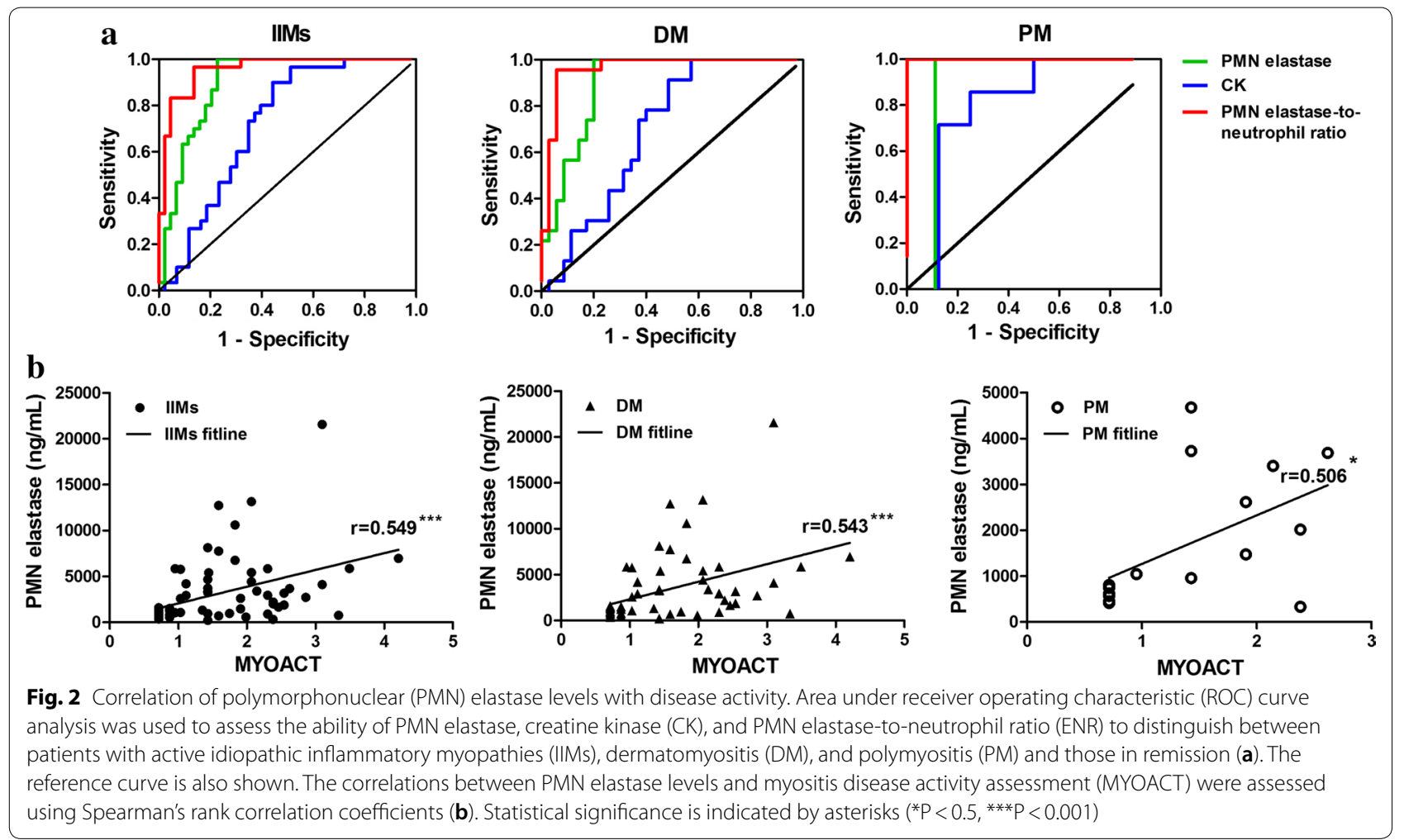

Table 2 AUC, PPV, NPV, sensitivity and specificity for PMN elastae in patients with myositis

\begin{tabular}{|c|c|c|c|c|c|c|c|c|c|}
\hline & IIMs & & & DM & & & PM & & \\
\hline & PMN elastase & CK & ENR & PMN elastase & CK & ENR & PMN elastase & CK & ENR \\
\hline $\begin{array}{l}\text { AUC } \\
(95 \% \mathrm{Cl})\end{array}$ & $\begin{array}{l}0.90^{* * *} \\
(0.83-0.97)\end{array}$ & $\begin{array}{l}0.72^{* *} \\
(0.61-0.84)\end{array}$ & $\begin{array}{l}0.96^{* * *} \\
(0.92-1.0)\end{array}$ & $\begin{array}{l}0.90^{* * *} \\
(0.82-0.98)\end{array}$ & $\begin{array}{l}0.70^{*} \\
(0.57-0.83)\end{array}$ & $\begin{array}{l}0.96^{* * *} \\
(0.91-1.0)\end{array}$ & $\begin{array}{l}0.88^{*} \\
(0.65-1.0)\end{array}$ & $\begin{array}{l}0.80^{*} \\
(0.56-1.0)\end{array}$ & $\begin{array}{l}1.0^{* *} \\
(1.0-1.0)\end{array}$ \\
\hline $\begin{array}{l}\text { Cut off value } \\
\text { (ng/mL) }\end{array}$ & 1632.4 & 84.8 & 329.0 & 1632.4 & 107.9 & 329.0 & 1632.4 & 107.9 & 329.0 \\
\hline PPV & 1 & 0.89 & 0.98 & 1 & 1 & 0.98 & 1 & 1 & 1 \\
\hline NPV & 0.77 & 0.59 & 0.85 & 0.68 & 0.44 & 0.89 & 0.78 & 0.58 & 1 \\
\hline Sensitivity (\%) & 79 & 56 & 88 & 80 & 42.9 & 94 & 80 & 43 & 94 \\
\hline Specificity (\%) & 100 & 90 & 97 & 100 & 100 & 96 & 100 & 100 & 96 \\
\hline
\end{tabular}

AUC area under the curve, PPV positive predictive value, NPV negative predictive value; $95 \%$ CI 95\% confidence interval, ENR PMN elastase-to-netrophil ratio

${ }^{*} \mathrm{P}<0.05$ for the area under the curve; ${ }^{* *} \mathrm{P}<0.01$ for the area under the curve; ${ }^{* *} \mathrm{P}<0.001$ for the area under the curve

emphasise the utility of PMN elastase as a potential biomarker for disease monitoring.

\section{PMN elastase-to-neutrophil ratio (ENR) value to distinguish active disease and remission}

Considering the diagnostic value of PMN elastase in distinguishing myositis patients with active disease and those with remission and the correlation of PMN elastase with neutrophil count (Table 3), we further investigated whether ENR, which is the ratio of serum PMN elastase level to serum neutrophil count, was correlated with disease activity. Results showed that ENR did not differ in patients with IIMs, DM and PM when compared with HCs (all P>0.05; Fig. 3a). In patients with IIMs with active disease, ENR was significantly higher than those in remission (751.5 (389.4-897.5) vs 146.3 (93.6-199.8), $\mathrm{P}<0.001$; Fig. 3b). After dividing the patients with IIMs into patients with DM and PM, both patient subgroups with active disease showed higher ENR than those in remission (776.1 (487.0-967.9) vs 151.8 (100.2-208.8), and $373.2(227.8-848.5)$ vs 97.8 (91.7-196.7), $\mathrm{P}<0.001$ and $\mathrm{P}=0.001$; Fig. $3 \mathrm{~b})$. ROC analysis was performed, and 
Table 3 Association of PMN elastase levels and ENR with laboratory parameters of patients with myositis

\begin{tabular}{|c|c|c|c|c|c|c|}
\hline & \multicolumn{3}{|c|}{ PMN elastase } & \multicolumn{3}{|l|}{ ENR } \\
\hline & IIMs & DM & PM & IIMs & DM & PM \\
\hline \multicolumn{7}{|l|}{ Muscle enzymes } \\
\hline CK & $0.308^{* *}$ & $0.339^{* *}$ & 0.229 & $0.289^{* *}$ & $0.332^{* *}$ & \\
\hline $\mathrm{LDH}$ & $0.366^{* *}$ & $0.386^{* *}$ & 0.211 & $0.287^{*}$ & $0.295^{*}$ & 0.307 \\
\hline ALT & $0.437^{* * *}$ & $0.406^{* * *}$ & 0.288 & $0.343^{* *}$ & $0.296^{*}$ & 0.251 \\
\hline AST & $0.438^{* * *}$ & $0.454^{* * *}$ & 0.23 & $0.419^{* * *}$ & $0.442^{* * *}$ & 0.197 \\
\hline \multicolumn{7}{|c|}{ Inflammation marker } \\
\hline CRP & $0.35^{* *}$ & $0.346^{*}$ & 0.194 & $0.329^{* *}$ & $0.357^{* *}$ & 0 \\
\hline ESR & $0.315^{* *}$ & $0.292^{* *}$ & 0.146 & $0.362^{* *}$ & $0.4^{* *}$ & 0.006 \\
\hline C4 & 0.11 & 0.02 & 0.168 & 0.159 & 0.121 & -0.077 \\
\hline C3 & -0.023 & -0.064 & 0.214 & 0.01 & 0.017 & -0.068 \\
\hline Neutrophil & $0.286^{* *}$ & $0.339^{* *}$ & 0.284 & -0.174 & -0.104 & -0.132 \\
\hline Lymphocyte & -0.275 & -0.279 & -0.314 & -0.388 & $-0.432^{* * *}$ & -0.241 \\
\hline NLR & $0.443^{* * *}$ & $0.485^{* *}$ & $0.361^{*}$ & $0.207^{*}$ & $0.291^{*}$ & 0.005 \\
\hline
\end{tabular}

Data were analysed using the Spearman's rank correlation

ENR PMN elastase-to-netrophil ratio, CK creatine phosphokinase, $A S T$ aspartate aminotransferase, $A L T$ alanine aminotransferase, $L D H$ lactate dehydrogenase, $C R P$ C-reactive protein, ESR erythrocyte sedimentation rate, C4 complement fraction 4, C3 complement fraction $3, N L R$ the ratio of neutrophil count to lymphocyte count

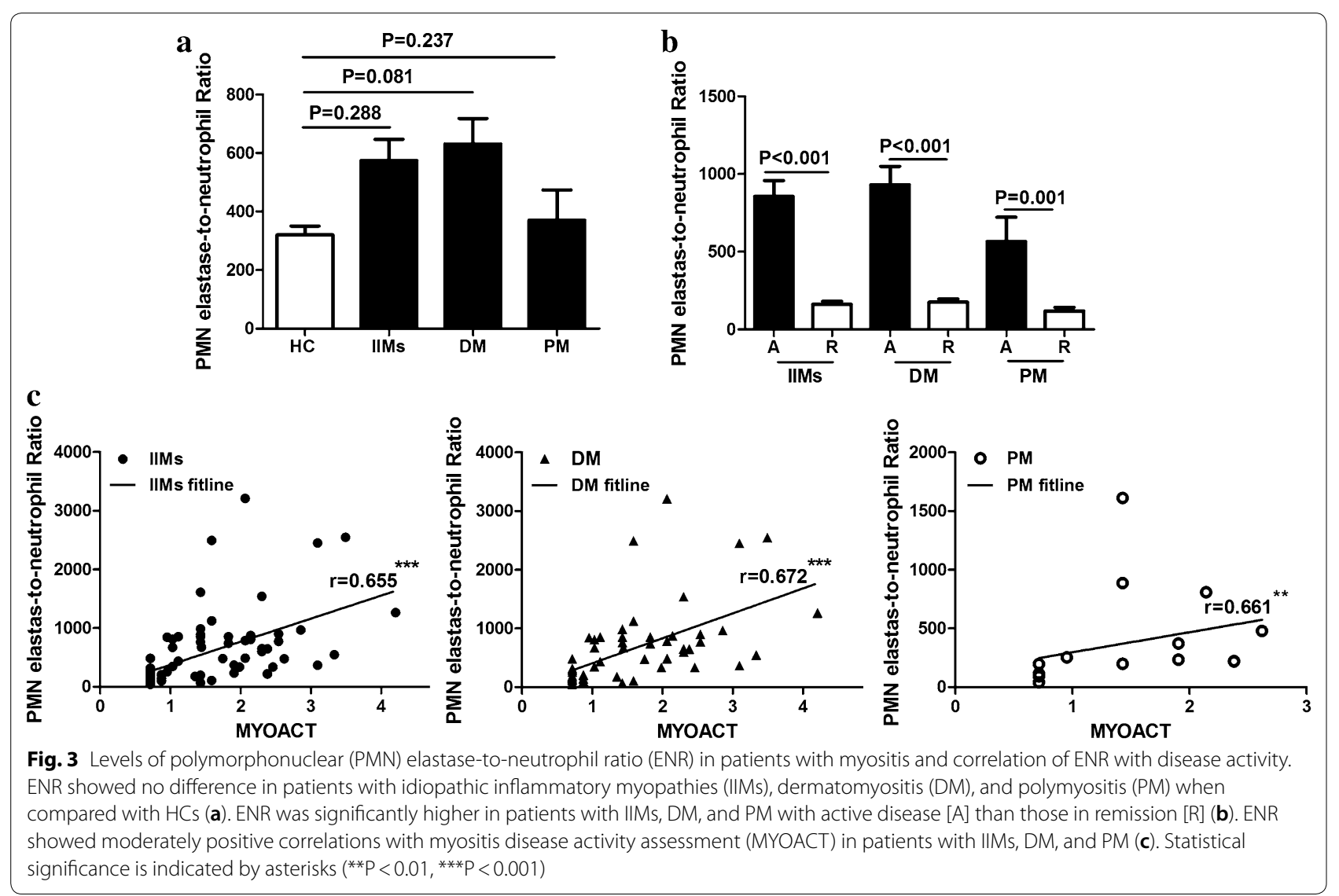

the ENR to distinguish active disease and remission had an AUC of 0.96 for IIMs, 0.96 for DM, and 1.0 for PM (Table 2). The sensitivity and specificity of ENR for active disease in IIMs were $88 \%$ and $97 \%$, and those in DM and PM were both $94 \%$ and $96 \%$, respectively (Table 2). Moreover, ENR was positively correlated with MYOACT, 
with $r$ values of 0.655 in IIMs $(\mathrm{P}<0.001$; Fig. $3 \mathrm{c}), 0.672$ in $\mathrm{DM}(\mathrm{P}<0.001$; Fig. $3 \mathrm{c})$, and 0.661 in $\mathrm{PM}(\mathrm{P}<0.01$; Fig. $3 \mathrm{c})$.

\section{Correlation of PMN elastase and ENR with muscle enzymes and inflammatory markers}

In patients with IIMs and DM, correlation analysis revealed that the serum PMN elastase level was positively associated with $\mathrm{CK}(\mathrm{r}=0.308$ and 0.339 , both $\mathrm{P}<0.01)$, LDH $(r=0.366$ and 0.386, both $\mathrm{P}<0.01)$, ALT $(r=0.437$ and 0.406 , both $\mathrm{P}<0.001$ ), and AST $(\mathrm{r}=0.438$ and 0.454 , both $\mathrm{P}<0.001)$. Furthermore, positive associations were found between PMN elastase and CRP ( $r=0.350$ and 0.346, $\mathrm{P}<0.01$ and $\mathrm{P}<0.05)$ and ESR levels $(\mathrm{r}=0.315$ and 0.292 , both $\mathrm{P}<0.01)$, neutrophil count $(\mathrm{r}=0.286$ and 0.339 , both $\mathrm{P}<0.01)$, and NLR $(\mathrm{r}=0.443$ and $0.485, \mathrm{P}<0.001$ and $\mathrm{P}<0.01$ ). No correlations were found for PMN elastase with C4 and C3 levels or lymphocyte count. In patients with PM, the positive association was only found between PMN elastase and NLR $(r=0.361, \mathrm{P}<0.05)$.

We also observed correlations between ENR and CK $(\mathrm{r}=0.289$ and 0.332 , both $\mathrm{P}<0.01)$, LDH $(\mathrm{r}=0.287$ and 0.295 , both $\mathrm{P}<0.05)$, ALT $(\mathrm{r}=0.343$ and $0.296, \mathrm{P}<0.01$ and $\mathrm{P}<0.05)$, AST $(\mathrm{r}=0.419$ and 0.442 , both $\mathrm{P}<0.001)$, CRP $(r=0.329$ and 0.357 , both $\mathrm{P}<0.01)$, ESR $(r=0.362$ and 0.400 , both $\mathrm{P}<0.01)$, and NLR $(\mathrm{r}=0.207$ and 0.291 , both $\mathrm{P}<0.05)$ in patients with IIMs and DM. ENR showed a moderately negative correlation with lymphocyte count $(\mathrm{r}=-0.432, \mathrm{P}<0.001)$ only in patients with DM. However, in patients with PM, no correlation was found between ENR and these indicators. Associations of PMN elastase levels and ENR with muscle enzymes and inflammation markers for each subgroup and all patients with myositis are listed in Table 3.
Association of PMN elastase and ENR with autoantibodies in patients with myositis

In patients with IIMs, the prevalence of ANA, antiRo-52, anti-MDA5, anti-Jo-1, anti-TIF-1 $\gamma$, anti-Mi-2, and anti-PM-Scl was 90\%, 55.4\%, 28.4\%, 20.3\%, 18.9\%, $14.9 \%$, and $10.8 \%$, respectively. The prevalence of other autoantibodies was all less than $10 \%$. We examined the difference in serum levels of PMN elastase and ENR between patents with and without elevation of an autoantibody. Our data showed that the serum levels of PMN elastase and ENR were higher in the anti-PM-Scl positive group than in the anti-PM-Scl negative group (5022.2 (2259.9-6684.8) vs 1445.8 (738.3-3404.8), ng/ $\mathrm{mL}, \mathrm{P}=0.007$, and $1127.4(866.8-2224.2)$ vs 317.5 (146.4-692.6), $\mathrm{P}<0.001$ ) in patients with IIMs, which was also observed in patients with DM. By contrast, no significant differences were observed between other autoantibody positive and negative groups. The prevalence of autoantibodies and association of PMN elastase and ENR with autoantibodies for each subgroup and for all patients with myositis are summarised in Table 4.

\section{Serum levels of PMN elastase and ENR in other autoimmune diseases}

To investigate whether PMN elastase and ENR were specifically elevated in myositis, PMN elastase levels and ENR in patients with SLE, SSc, and RA were measured. Results showed that the PMN elastase levels were slightly elevated in patients with RA and those with SSc $(P=0.036$ and 0.031 ; Fig. 4a), but not in patients with SLE ( $\mathrm{P}>0.05$; Fig. 4a). After dividing the patients into active and remission states, the PMN

Table 4 The associations of PMN elastase and ENR with autoantibodies in myositis patients

\begin{tabular}{|c|c|c|c|c|c|c|c|c|c|c|c|c|}
\hline & \multicolumn{2}{|l|}{ IIMs } & \multicolumn{2}{|l|}{ P value } & \multicolumn{2}{|l|}{ DM } & \multicolumn{2}{|l|}{ P value } & \multicolumn{2}{|l|}{ PM } & \multicolumn{2}{|l|}{ P value } \\
\hline & $\mathrm{P} / \mathrm{N}$ & $\%$ & PMN elastase & ENR & $\mathrm{P} / \mathrm{N}$ & $\%$ & PMN elastase & ENR & $\mathrm{P} / \mathrm{N}$ & $\%$ & PMN elastase & ENR \\
\hline ANA & $63 / 7$ & 90.0 & 0.619 & 0.724 & $47 / 7$ & 87.0 & 0.413 & 0.489 & $16 / 0$ & 100 & - & - \\
\hline Anti-Ro-52 & $41 / 33$ & 55.4 & 0.732 & 0.511 & $28 / 30$ & 48.3 & 0.250 & 0.109 & $13 / 3$ & 81.3 & 0.638 & 0.545 \\
\hline Anti-Jo-1 & $15 / 59$ & 20.3 & 0.633 & 0.386 & $7 / 51$ & 12.1 & 0.072 & 0.467 & $8 / 8$ & 100 & 0.916 & 0.401 \\
\hline Anti-PM-Scl & $8 / 66$ & 10.8 & 0.007 & 0.001 & $8 / 50$ & 16.0 & 0.013 & 0.001 & $0 / 16$ & 0 & - & - \\
\hline Anti-MDA5 & $21 / 53$ & 28.4 & 0.619 & 0.385 & $21 / 37$ & 36.2 & 0.289 & 0.740 & $0 / 16$ & 0 & - & - \\
\hline Anti-Mi-2 & $11 / 63$ & 14.9 & 0.184 & 0.074 & $11 / 47$ & 19.0 & 0.326 & 0.124 & $0 / 16$ & 0 & - & - \\
\hline Anti-TIF-1Y & $14 / 60$ & 18.9 & 0.649 & 0.610 & $12 / 46$ & 20.1 & 0.618 & 0.420 & $2 / 14$ & 12.5 & 0.056 & 0.056 \\
\hline Anti-NXP2 & $4 / 70$ & 5.4 & 0.830 & 0.793 & $3 / 55$ & 5.2 & 0.352 & 0.562 & $1 / 15$ & 6.3 & 0.159 & 0.159 \\
\hline Anti-PL7 & $4 / 70$ & 5.4 & 0.056 & 0.054 & $4 / 54$ & 6.9 & 0.08 & 0.07 & $0 / 16$ & 0 & - & - \\
\hline Anti-Ku & $2 / 72$ & 2.7 & 0.334 & 0.443 & $1 / 57$ & 1.7 & 0.179 & 0.16 & $1 / 15$ & 6.3 & 0.745 & 0.745 \\
\hline Anti-EJ & $4 / 70$ & 5.4 & 0.304 & 0.189 & $1 / 57$ & 1.7 & 0.128 & 0.269 & $3 / 13$ & 18.8 & 0.737 & 0.946 \\
\hline Anti-SAE1 & $3 / 71$ & 4.1 & 0.184 & 0.064 & $3 / 55$ & 5.2 & 0.136 & 0.056 & $0 / 16$ & 0 & - & - \\
\hline Anti-SRP & $3 / 71$ & 4.1 & 0.077 & 0.150 & $1 / 57$ & 1.7 & 0.324 & 0.570 & $2 / 14$ & 12.5 & 0.266 & 0.266 \\
\hline
\end{tabular}


elastase levels were moderately higher in patients with active SLE and SSc than those in remission $(\mathrm{P}=0.049$ and 0.041; Fig. 4b).

Moreover, the ENR showed no differences in patients with SLE, SSc, and RA, when compared with HCs (all $\mathrm{P}>0.05$; Fig. $4 \mathrm{c}$ ). However, after dividing the patients into active and remission states, the ENR in patients with active SLE and SSc was higher than those in remission $(P=0.005$ and 0.019 , respectively; Fig. $4 d)$.

\section{Discussion}

In this study, the level of PMN elastase was significantly increased in patients with IIMs and was positively correlated with clinical indicators, such as CK, LDH, ALT, AST, ESR, CRP, neutrophil count, and NLR. Importantly, PMN elastase strongly distinguished between IIM patients with active disease and those in remission. Moreover, we reported a new indicator, ENR. This indicator did not show increased levels in patients with IIMs when compared with $\mathrm{HCs}$, but was superior to PMN elastase in evaluating the disease activity of patients with IIMs. This study is the first to focus on PMN elastase and
ENR in distinguishing between active IIMs and IIMs in remission.

PMN elastase is a serine proteinase that is mainly stored in the azurophil granules of neutrophils [32]. When these cells are stimulated by microorganisms or other invading pathogens, PMN elastase is released into the extracellular space and exerts several effects, such as processing inflammatory mediators and combining with oxidants. Our results showed that PMN elastase levels were significantly higher in patients with IIMs and DM, which was consistent with the findings of the study carried out by Gao et al. [23]. In the total PM patient group, the PMN elastase level was not significantly altered when compared to that in healthy donors, but was significantly higher in those with active disease than those in remission. The reasons for the negative result between the PM patient group and healthy donors may due to the data variation between different PM patients, particularly those in remission, and the limited sample numbers of PM patients. We further compared the PMN elastase level between active PM patients and healthy donors; this showed that active PM patients have higher PMN

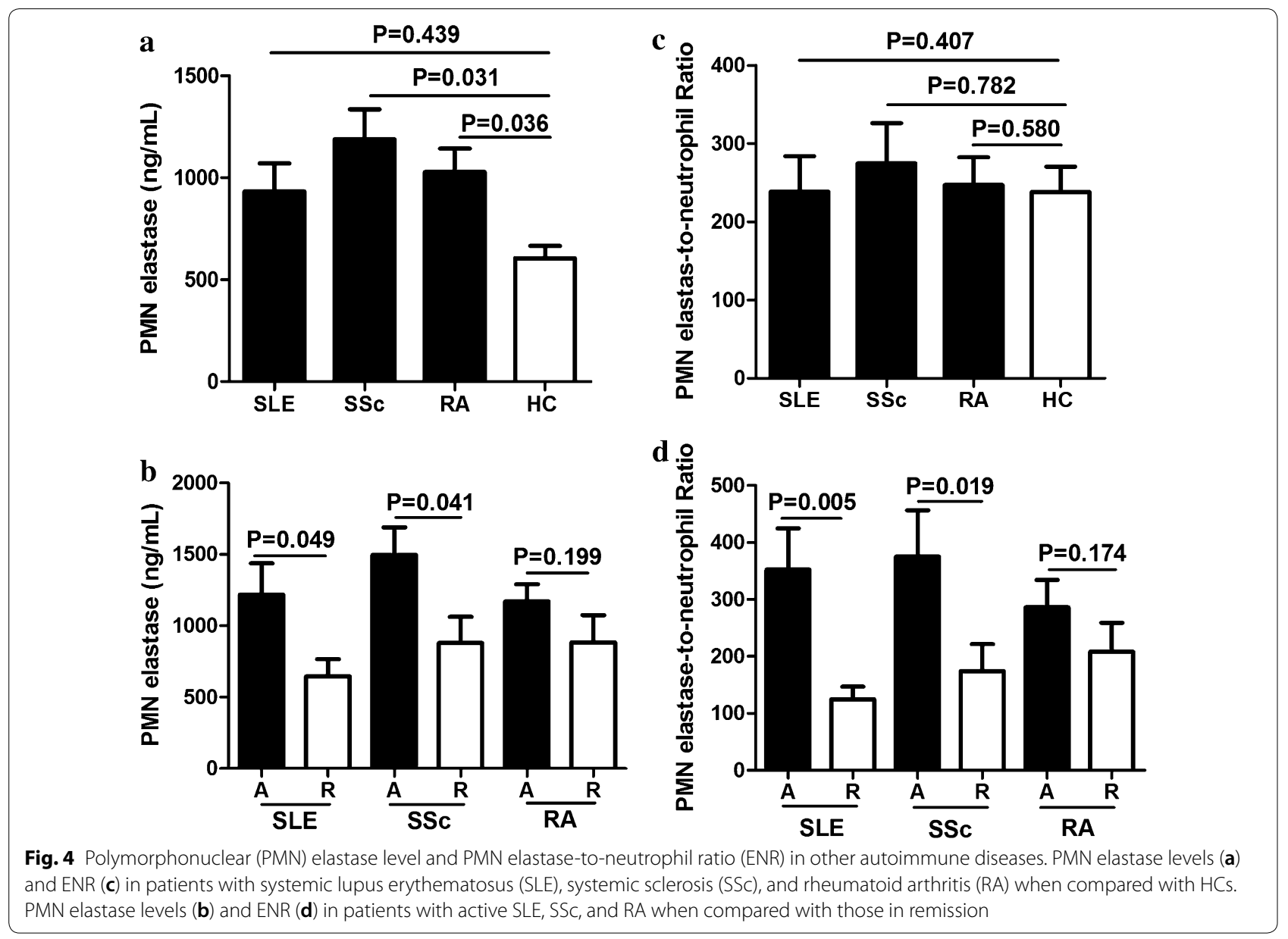


elastase levels than healthy donors $(\mathrm{P}=0.03$, data not shown), which confirmed our supposition.

Previous studies also showed that in patients with RA [33] and SSc [34], the serum PMN elastase levels are elevated, and the activity of PMN elastase is increased in destructive joints in RA. These findings were similar to our results. However, no differences were found in patients with SLE, which may be due to the limited number of samples; thus, further investigation is warranted. These results suggest that during systemic inflammatory states or in autoimmune diseases, the serum PMN elastase level could increase. Interestingly, the PMN elastase levels were higher in patients with IIMs than those in patients with other autoimmune diseases in our study cohort. While the reasons for this difference are not clear, it might be related to the different pathogenetic mechanisms involved in the different diseases.

ENR, a new indicator reported first in this study, is the ratio of serum PMN elastase level to serum neutrophil count, representing the average level of elastase in each PMN cell. Plasma PMN elastase originates mainly from PMN cells and indicates the activity of PMN. Although the role of PMN elastase in inflammatory diseases has been partially investigated, data on ENR in inflammation are not available in the literature. We found that ENR did not increase in patients with IIMs, RA, SSc, and SLE when compared with $\mathrm{HCs}$, but was significantly higher in patients with active IIM, SSc, and SLE than those in remission. The PMN elastase levels in patients with IIMs, $\mathrm{RA}$, and SSc were higher than those in controls; PMN neutrophil counts in these diseases were correspondingly increased. PMN elastase levels were much higher in active disease than those in remission, but PMN neutrophil counts showed no difference between these two states. This indicates that PMN neutrophil counts are not reduced to basal levels in the remission state, and that PMN elastase levels not only correlate with PMN neutrophil counts, but also with neutrophil activity.

Our results show that both PMN elastase level and ENR may be useful biochemical markers for evaluating the disease activity of patients with IIMs. A study by Asim et al. revealed that in patients with psoriasis, PMN elastase may be a good marker for diagnosis and follow up of the disease activity, but the evidence in evaluating disease activity was limited [20]. Here, we propose this conclusion based on the following three points: first, PMN elastase level and ENR were significantly higher in patients with active IIMs than those in remission. Second, we found that serum PMN elastase level and ENR were positively correlated with MYOACT, CK, LDH, ALT, AST, CRP, and ESR. MYOACT is a tool used to assess myositis disease activity, and serum CK, $\mathrm{LDH}, \mathrm{ALT}$, and AST levels have been reported to have moderate correlation with muscle weakness and inflammation, which tend to indicate disease activity $[5,6,35]$. Serum levels of ESR and CRP are risk factors for ILD in DM/PM [36]. Third, the ROC curve analyses greatly supported our conclusions. PMN elastase level and ENR represent good bases for distinguishing between active and remission IIMs, with an AUC of 0.9 and 0.96. ENR was superior to PMN elastase in evaluating the disease activity of patients with IIMs. Furthermore, both biomarkers outperformed CK, which is commonly used as a laboratory marker for disease activity and is one of the current criteria for determining clinically inactive disease in juvenile DM $[37,38]$. All these findings indicate that PMN elastase and ENR are potential markers for estimating disease activity in IIMs.

Current treatment guidelines recommend that immunosuppression is the traditional first-line treatment for patients with myositis, but treatment duration varies in different patients [39]. For example, some patients may benefit from shorter treatment duration; overtreatment with steroids can result in serious side effects. Hence, objective measurement of disease activity is crucial to determine the rate of medication tapering and to avoid both under- and overtreatment. Despite the novel and clinically relevant findings in this study, there are some limitations. The patients included in our study differed in severity and had different treatment options. Larger sample size and multi-cohort validation are needed. Thus, further studies should be performed to confirm our conclusion and promote this clinical application.

\section{Conclusion}

This study is the first to show that PMN elastase and particularly ENR significantly correlate with disease activity. Thus, they might serve as biomarkers in patients with IIMs. Even in this small study cohort, the power of these markers to discriminate between patients with active disease and those in remission was highly significant, which emphasises their potential as biomarkers to monitor disease and guide treatment. Determination of the clinical value of PMN elastase and ENR is warranted in future prospective studies.

\footnotetext{
Abbreviations

ALT: alanine aminotransferase; ANA: antinuclear antibody; AST: aspartate aminotransferase; AUC: area under the ROC curve; C3: complement fractions 3; C4: complement fractions 4; CK: creatine kinase; CRP: C-reactive protein; DM: dermatomyositis; ENR: elastase-to-neutrophil ratio; ESR: erythrocyte sedimentation rate; $\mathrm{HC}$ : healthy control; IIM: idiopathic inflammatory myopathies; ILD: interstitial lung disease; LDH: lactate dehydrogenase; MYOACT: myositis disease activity assessment; NLR: neutrophil to lymphocyte ratio; PM: polymyositis; PMN: polymorphonuclear; RA: rheumatoid arthritis; ROC: receiver operating characteristic; SLE: systemic lupus erythematosus; SSc: systemic sclerosis.
} 


\section{Acknowledgements \\ Not applicable.}

\section{Authors' contributions}

WW designed the experiments; WSY and PWC performed the experiments; WSY analysed the data and wrote the paper. All authors read and approved the final manuscript.

\section{Funding}

This study was supported by the "National Natural Science Foundation of China" (Grant No. 81401731)

\section{Availability of data and materials}

All data generated or analysed during this study are included in this published article. If any additional information is required it may be obtained by request with the corresponding author.

\section{Ethics approval and consent to participate}

Informed written consent was obtained from all subjects, and the study was approved by the Ethics Committee of Xiangya Hospital, Central South University.

\section{Consent for publication}

Not applicable.

\section{Competing interests}

The authors declare that they have no competing interests.

Received: 3 September 2019 Accepted: 10 December 2019 Published online: 16 December 2019

\section{References}

1. Dalakas MC, Sivakumar K. The immunopathologic and inflammatory differences between dermatomyositis, polymyositis and sporadic inclusion body myositis. Curr Opin Neurol. 1996;9(3):235-9.

2. Rayavarapu S, Coley W, Kinder TB, Nagaraju K. Idiopathic inflammatory myopathies: pathogenic mechanisms of muscle weakness. Skelet Muscle. 2013;3(1):13.

3. Luo YB, Mastaglia FL. Dermatomyositis, polymyositis and immune-mediated necrotising myopathies. Biochim Biophys Acta. 2015;1852(4):622-32.

4. Gao S, Luo H, Zhang H, Zuo X, Wang L, Zhu H. Using multi-omics methods to understand dermatomyositis/polymyositis. Autoimmun Rev. 2017:16(10):1044-8

5. Rider LG. Assessment of disease activity and its sequelae in children and adults with myositis. Curr Opin Rheumatol. 1996;8(6):495-506.

6. Volochayev R, Csako G, Wesley R, Rider LG, Miller FW. Laboratory test abnormalities are common in polymyositis and dermatomyositis and differ among clinical and demographic groups. Open Rheumatol J. 2012;6:54-63.

7. Rider LG, Werth VP, Huber AM, Alexanderson H, Rao AP, Ruperto N, Herbelin L, Barohn R, Isenberg D, Miller FW. Measures of adult and juvenile dermatomyositis, polymyositis, and inclusion body myositis: physician and Patient/Parent Global Activity, Manual Muscle Testing (MMT), Health Assessment Questionnaire (HAQ)/Childhood Health Assessment Questionnaire (C-HAQ), Childhood Myositis Assessment Scale (CMAS), Myositis Disease Activity Assessment Tool (MDAAT), Disease Activity Score (DAS), Short Form 36 (SF-36), Child Health Questionnaire (CHQ), physician global damage, Myositis Damage Index (MDI), Quantitative Muscle Testing (QMT), Myositis Functional Index-2 (Fl-2), Myositis Activities Profile (MAP), Inclusion Body Myositis Functional Rating Scale (IBMFRS), Cutaneous Dermatomyositis Disease Area and Severity Index (CDASI), Cutaneous Assessment Tool (CAT), Dermatomyositis Skin Severity Index (DSSI), Skindex, and Dermatology Life Quality Index (DLQI). Arthritis Care Res (Hoboken). 2011;63(Suppl 11):S118-57.

8. Aggarwal R, Rider LG, Ruperto N, Bayat N, Erman B, Feldman BM, Oddis CV, Amato AA, Chinoy H, Cooper RG, et al. 2016 American College of Rheumatology/European League Against Rheumatism Criteria for Minimal, Moderate, and Major Clinical Response in Adult Dermatomyositis and Polymyositis: an International Myositis Assessment and Clinical Studies Group/Paediatric Rheumatology International Trials Organisation Collaborative Initiative. Arthritis Rheumatol. 2017;69(5):898-910.

9. Bellutti Enders F, van Wijk F, Scholman R, Hofer M, Prakken BJ, van Royen-Kerkhof A, de Jager W. Correlation of CXCL10, tumor necrosis factor receptor type $\mathrm{I}$, and galectin 9 with disease activity in juvenile dermatomyositis. Arthritis Rheumatol. 2014;66(8):2281-9.

10. Wienke J, Bellutti Enders F, Lim J, Mertens JS, van den Hoogen LL, Wijngaarde CA, Yeo JG, Meyer A, Otten HG, Fritsch-Stork RDE, et al. Galectin-9 and CXCL10 as biomarkers for disease activity in juvenile dermatomyositis: a longitudinal cohort study and multicohort validation. Arthritis Rheumatol. 2019;71(8):1377-90.

11. Andres Cerezo L, Hulejova H, Sumova B, Kropackova T, Krystufkova O, Klein M, Mann HF, Zamecnik J, Pecha O, Pavelka K, et al. Pro-inflammatory S100A 11 is elevated in inflammatory myopathies and reflects disease activity and extramuscular manifestations in myositis. Cytokine. 2019;116:13-20.

12. Zhou H, Wang Y, Bi K, Qi H, Song S, Zhou M, Chen L, Wang G, Duan T. Serum-soluble TRAIL: a potential biomarker for disease activity in myositis patients. Clin Rheumatol. 2019:38(5):1425-31.

13. Gao MZ, Wei YY, Xu QW, Ji R, Han ZJ, Jiang TW. Elevated serum YKL-40 correlates with clinical characteristics in patients with polymyositis or dermatomyositis. Ann Clin Biochem. 2019;56(1):95-9.

14. Yang Y, Liu Y, Huang L, Wang L, Liu K, Liu M, Luo H, Zuo X, Li Y, Zhang $H$. Clinical features and cytokine profile in myositis patients with antiEJ autoantibodies detected by a novel immunoprecipitation assay. Biomed Res Int. 2019:2019:1856180.

15. Korkmaz B, Horwitz MS, Jenne DE, Gauthier F. Neutrophil elastase, proteinase 3, and cathepsin $\mathrm{G}$ as therapeutic targets in human diseases. Pharmacol Rev. 2010;62(4):726-59.

16. Muley MM, Reid AR, Botz B, Bolcskei K, Helyes Z, McDougall JJ. Neutrophil elastase induces inflammation and pain in mouse knee joints via activation of proteinase-activated receptor-2. Br J Pharmacol. 2016;173(4):766-77.

17. Langhorst J, Elsenbruch S, Koelzer J, Rueffer A, Michalsen A, Dobos GJ. Noninvasive markers in the assessment of intestinal inflammation in inflammatory bowel diseases: performance of fecal lactoferrin, calprotectin, and PMN-elastase, CRP, and clinical indices. Am J Gastroenterol. 2008; 103(1):162-9.

18. Langhorst J, Boone J, Lauche R, Rueffer A, Dobos G. Faecal lactoferrin calprotectin, PMN-elastase, CRP, and white blood cell count as indicators for mucosal healing and clinical course of disease in patients with mild to moderate ulcerative colitis: post hoc analysis of a prospective clinical trial. J Crohns Colitis. 2016;10(7):786-94.

19. Kawabata K, Hagio T, Matsuoka S. The role of neutrophil elastase in acute lung injury. Eur J Pharmacol. 2002;451(1):1-10.

20. Orem A, Deger O, Cimsit G, Bahadir S. Plasma polymorphonuclear leukocyte elastase levels and its relation to disease activity in psoriasis. Clin Chim Acta. 1997:264(1):49-56.

21. Shioya Y, Katakura K, Ohira H. Neutrophil elastase inhibitor suppresses IL-17 based inflammation of murine experimental colitis. Fukushima J Med Sci. 2014;60(1):14-21.

22. Ferreira AV, Perelshtein I, Perkas N, Gedanken A, Cunha J, Cavaco-Paulo A. Detection of human neutrophil elastase (HNE) on wound dressings as marker of inflammation. Appl Microbiol Biotechnol. 2017;101(4):1443-54.

23. Gao S, Zuo X, Liu D, Xiao Y, Zhu H, Zhang H, Luo H. The roles of neutrophil serine proteinases in idiopathic inflammatory myopathies. Arthritis Res Ther. 2018;20(1):134.

24. Gao S, Zhu H, Yang H, Zhang H, Li Q, Luo H. The role and mechanism of cathepsin G in dermatomyositis. Biomed Pharmacother. 2017:94:697-704

25. Bohan A, Peter JB. Polymyositis and dermatomyositis (first of two parts). N Engl J Med. 1975;292(7):344-7.

26. Bohan A, Peter JB. Polymyositis and dermatomyositis (second of two parts). N Engl J Med. 1975;292(8):403-7.

27. Isenberg DA, Allen E, Farewell V, Ehrenstein MR, Hanna MG, Lundberg IE, Oddis C, Pilkington C, Plotz P, Scott D, et al. International consensus outcome measures for patients with idiopathic inflammatory myopathies Development and initial validation of myositis activity and damage indices in patients with adult onset disease. Rheumatology (Oxford). 2004;43(1):49-54 
28. Shu XM, Lu X, Xie Y, Wang GC. Clinical characteristics and favorable longterm outcomes for patients with idiopathic inflammatory myopathies: a retrospective single center study in China. BMC Neurol. 2011;11:143.

29. Yee CS, Farewell VT, Isenberg DA, Griffiths B, Teh LS, Bruce IN, Ahmad Y, Rahman A, Prabu A, Akil M, et al. The use of Systemic Lupus Erythematosus Disease Activity Index-2000 to define active disease and minimal clinically meaningful change based on data from a large cohort of systemic lupus erythematosus patients. Rheumatology (Oxford). 2011;50(5):982-8.

30. Valentini G, ludici M, Walker UA, Jaeger VK, Baron M, Carreira P, Czirjak L, Denton CP, Distler O, Hachulla E, et al. The European Scleroderma Trials and Research group (EUSTAR) task force for the development of revised activity criteria for systemic sclerosis: derivation and validation of a preliminarily revised EUSTAR activity index. Ann Rheum Dis. 2017;76(1):270-6.

31. Fleischmann RM, van der Heijde D, Gardiner PV, Szumski A, Marshall L, Bananis E. DAS28-CRP and DAS28-ESR cut-offs for high disease activity in rheumatoid arthritis are not interchangeable. RMD Open. 2017;3(1):e000382.

32. Owen CA, Campbell EJ. The cell biology of leukocyte-mediated proteolysis. J Leukoc Biol. 1999;65(2):137-50.

33. Momohara S, Kashiwazaki S, Inoue K, Saito S, Nakagawa T. Elastase from polymorphonuclear leukocyte in articular cartilage and synovial fluids of patients with rheumatoid arthritis. Clin Rheumatol. 1997;16(2):133-40.

34. Hara T, Ogawa F, Yanaba K, Iwata Y, Muroi E, Komura K, Takenaka M, Shimizu K, Hasegawa M, Fujimoto M, et al. Elevated serum concentrations of polymorphonuclear neutrophilic leukocyte elastase in systemic sclerosis: association with pulmonary fibrosis. J Rheumatol. 2009;36(1):99-105.
35. Lundberg IE, Tjarnlund A, Bottai M, Werth VP, Pilkington C, Visser M, Alfredsson L, Amato AA, Barohn RJ, Liang MH, et al. 2017 European League Against Rheumatism/American College of Rheumatology classification criteria for adult and juvenile idiopathic inflammatory myopathies and their major subgroups. Ann Rheum Dis. 2017;76(12):1955-64.

36. Zhang L, Wu G, Gao D, Liu G, Pan L, Ni L, Li Z, Wang Q. Factors associated with interstitial lung disease in patients with polymyositis and dermatomyositis: a systematic review and meta-analysis. PLOS ONE. 2016;11(5):e0155381.

37. Lazarevic D, Pistorio A, Palmisani E, Miettunen P, Ravelli A, Pilkington C, Wulffraat NM, Malattia C, Garay SM, Hofer M, et al. The PRINTO criteria for clinically inactive disease in juvenile dermatomyositis. Ann Rheum Dis. 2013;72(5):686-93.

38. Almeida B, Campanilho-Marques R, Arnold K, Pilkington CA, Wedderburn LR, Nistala K. Juvenile dermatomyositis research $g$ analysis of published criteria for clinically inactive disease in a large juvenile dermatomyositis cohort shows that skin disease is underestimated. Arthritis Rheumatol. 2015;67(9):2495-502.

39. Needham M, Mastaglia FL. Immunotherapies for immune-mediated myopathies: a current perspective. Neurotherapeutics. 2016;13(1):132-46.

\section{Publisher's Note}

Springer Nature remains neutral with regard to jurisdictional claims in published maps and institutional affiliations.
Ready to submit your research? Choose BMC and benefit from:

- fast, convenient online submission

- thorough peer review by experienced researchers in your field

- rapid publication on acceptance

- support for research data, including large and complex data types

- gold Open Access which fosters wider collaboration and increased citations

- maximum visibility for your research: over $100 \mathrm{M}$ website views per year

At BMC, research is always in progress.

Learn more biomedcentral.com/submissions 